\title{
Large-scale Phase-field Studies of Three-dimensional Dendrite Competitive Growth at the Converging Grain Boundary during Directional Solidification of a Bicrystal Binary Alloy
}

\author{
Tomohiro TAKAKI, ${ }^{1) *}$ Shinji SAKANE, ${ }^{2)}$ Munekazu OHNO, ${ }^{3)}$ Yasushi SHIBUTA, ${ }^{4)}$ Takashi SHIMOKAWABE ${ }^{5)}$ \\ and Takayuki AOKI ${ }^{5)}$
}

1) Faculty of Mechanical Engineering, Kyoto Institute of Technology, Matsugasaki, Sakyo-ku, Kyoto, 606-8585 Japan.

2) Graduate School of Science and Technology, Kyoto Institute of Technology, Matsugasaki, Sakyo-ku, Kyoto, 606-8585 Japan. 3) Division of Materials Science and Engineering, Faculty of Engineering, Hokkaido University, Kita 13 Nishi 8 , Kita-ku, Sapporo, 060-8628 Japan. $\quad 4)$ Department of Materials Engineering, The University of Tokyo, 7-3-1 Hongo, Bunkyo-ku, Tokyo, 113-8656 Japan. $\quad 5)$ Global Scientific Information and Computing Center, Tokyo Institute of Technology, 2-12-1-i7-3, Ohokayama, Meguro-ku, Tokyo, 152-8550 Japan.

(Received on March 17, 2016; accepted on May 12, 2016; J-STAGE Advance published date: June 23, 2016)

\begin{abstract}
Large-scale phase-field studies of three-dimensional (3D) dendrite competitive growth at the converging grain boundary (GB) of a bicrystal binary alloy were carried out using the GPU-rich supercomputer TSUBAME 2.5 at Tokyo Institute of Technology. First, a series of thin-sample simulations were performed to investigate the effects of thin-sample thickness, unfavorably oriented (UO) grain inclination angle, and dendrite arrangement on an unusual overgrowth phenomenon whereby the favorably oriented (FO) grain is overgrown by the $\cup O$ grain. It was concluded that the unusual overgrowth easily occurs as the thickness of the thin sample and the UO grain inclination angle decrease. It was also concluded that the interaction between FO and UO dendrites at the converging GB depends on the dendrite arrangement for relatively large dendrite spacing. Next, realistic large-scale simulations whereby multiple dendrites interact at the converging GB were performed. Unusual overgrowth was also observed in such large-scale simulations, and this phenomenon easily occurred at smaller $U O$ dendrite inclination angles. Furthermore, it was also concluded that the $\mathrm{FO}$ and $\mathrm{UO}$ dendrites rearrange toward a space-to-face interaction. Because the interaction between FO and UO dendrites differs according to the location on the GB, a zigzag GB was formed, especially at small UO grain inclination angles.
\end{abstract}

KEY WORDS: phase-field method; large-scale computation; directional solidification; competitive growth; dendrite.

\section{Introduction}

In casting, solidification is usually initiated from the chilled mold surface, and a columnar structure is formed through grain selection. ${ }^{1)}$ The Walton-Chalmers model ${ }^{2)}$ is widely accepted as a general competitive-growth model of grain selection. In this model, favorably oriented (FO) grains, for which the angle between the preferred growth direction and the heat-flow direction is small, can keep growing by blocking unfavorably oriented (UO) grains, which have a larger inclination angle than the FO grains. ${ }^{3-5}$ )

Recently, in contrast to the Walton-Chalmers model, an unusual overgrowth, whereby the UO grain overgrows the FO grain at the converging grain boundary (GB), has been reported in the case of directional solidification of bicrystals of a Ni-based superalloy ${ }^{6-8)}$ and succinonitrileacetone alloy. ${ }^{9)}$ To clarify the mechanism of this unusual

* Corresponding author: E-mail: takaki@kit.ac.jp DOI: http://dx.doi.org/10.2355/isijinternational.ISIJINT-2016-156 overgrowth, two-dimensional (2D) phase-field simulations of directional solidification of a bicrystal binary alloy were performed. ${ }^{10-13)}$ When the UO dendrite approaches the FO dendrite at the converging GB, the UO dendrite is usually overgrown by the FO dendrite owing to the lag of the tip position in the heat-flow direction. At the same time, owing to the solute interaction around the tips of FO and UO dendrites, the FO dendrite is pushed in the direction of the next FO dendrite. As this process is repeated, the spacing between the FO dendrite at the converging GB and the neighboring FO dendrite is gradually reduced; finally, the FO dendrite at the converging GB is overgrown when the spacing between two FO dendrites reaches a critical value. ${ }^{11,12)}$ This unusual overgrowth is frequently observed in 2D polycrystal grain competitive growth. ${ }^{14)}$

Grain selection in three-dimensional (3D) space is much more complicated than that in 2D space. ${ }^{15,16)}$ Unlike the $2 \mathrm{D}$ case, the interaction between FO and UO dendrites in the 3D case is not necessarily face-to-face, and there is a nonuniplanar GB, where the FO and UO dendrites are in a twist- 
ing relation, in addition to the converging and diverging GBs. ${ }^{17)}$ The above-mentioned unusual overgrowth has also been observed in 3D bicrystal competitive growth. ${ }^{17-19)}$ It is important to clarify 3D grain selection behavior for metallurgy applications and directional solidification products, such as turbine blades. ${ }^{20-22)}$

At present, the phase-field method is the only known method for accurately simulating the dendrite structure during solidification. ${ }^{15,23-25)}$ However, 3D phase-field simulation of multiple dendrites remains a challenging task owing to the high computational cost, in spite of the development of quantitative phase-field models. ${ }^{26-31)}$ Therefore, there is an urgent need for a large-scale simulation technique to facilitate future phase-field studies. Computation using a graphical processing unit (GPU) is a promising approach in the field of computational materials science. ${ }^{13,30,32-35)}$ Parallelization of multiple GPUs has accelerated large-scale phase-field simulations. ${ }^{11,12,14-16,36-38)}$

In this study, we perform large-scale phase-field simulations to investigate 3D unusual overgrowth at the converging GB during directional solidification of a bicrystal binary alloy. First, we perform a series of thin-sample simulations and investigate the effects of sample thickness, UO grain inclination angle, and dendrite arrangement on the unusual overgrowth. Here, we can observe an ideal one-to-one interaction between FO and UO dendrites, as an extension of our previous $2 \mathrm{D}$ investigation. ${ }^{11)} \mathrm{Next}$, we perform very-largescale simulations to investigate the realistic interactions among multiple FO and UO dendrites at the converging GB. Through these very-large-scale simulations, we can examine what happens in the realistic system as observed in the experiments ${ }^{17-19)}$ and clarify the difference between the realistic multiple dendrite interaction system and the ideal 2D and thin-sample systems. In these simulations, we employed the GPU-rich supercomputer TSUBAME 2.5 at Tokyo Institute of Technology.

\section{Numerical Simulations}

A quantitative phase-field model of dilute binary alloy solidification, developed by Ohno and Matsuura, ${ }^{29)}$ is applied to 3D directional solidification of a bicrystal. Time evolution equations for the phase-field variable $\phi$, which is defined as $\phi=1$ in the solid phase and $\phi=-1$ in the liquid phase, and the nondimensional supersaturation $u=$ $\left(c_{l}-c_{l}^{e}\right) /\left(c_{l}^{e}-c_{s}^{e}\right)$, where $c_{l}$ is the concentration in the liquid phase, and $c_{l}^{e}$ and $c_{s}{ }^{e}$ are the equilibrium concentrations in the liquid and solid phases, respectively, are solved numerically using the finite difference method. Regarding the temperature $T$, we assume a frozen temperature approximation $^{39,40)}$ where $T$ changes as $T(z)=T_{0}+G\left(z-V_{p} t\right)$, where, $z$ is the coordinate in the heat-flow direction, $T_{0}$ is the reference temperature at $z=0$ and $t=0, G$ is the temperature gradient, and $V_{p}$ is the pulling velocity. The details of the governing equations, discretization method, and parallel-GPU computation scheme have been provided in our previous paper. ${ }^{36)}$

Figure 1 shows the computational domain, which is meshed by $n_{x} \times n_{y} \times n_{z}$ lattice points. A zero Neumann boundary condition at the $x-y$ and $z-x$ surfaces and a periodic boundary condition at the $y-z$ surface are imposed for $\phi$ and

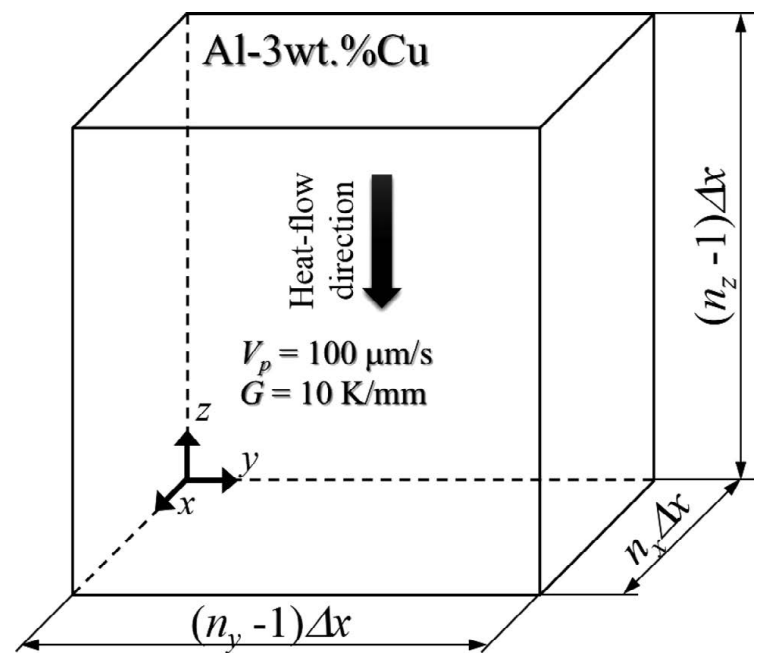

Fig. 1. Computational domain used in this study. Here, $n_{x}, n_{y}$, and $n_{z}$ are the number of lattice points in the $x$-, $y$-, and $z$-directions, respectively.

$u$. The actual domain size becomes $n_{x} \Delta x \times\left(n_{y}-1\right) \Delta x \times\left(n_{z}-1\right)$ $\Delta x$ with a mesh size $\Delta x$. The heat-flow direction is set to the $z$-direction. A binary alloy of $\mathrm{Al}-3 \mathrm{wt} \% \mathrm{Cu}$ is used as a sample material. Initially, the computational domain is filled with $\mathrm{Al}-3 \mathrm{wt} \% \mathrm{Cu}$ liquid $(\phi=-1)$, and undercooling $u_{0}=$ -0.3 is set to the bottom surface $(z=0)$. Figure 2 shows the initial solid seed arrangements at the bottom surface. In a series of thin-sample simulations, two types of seed arrangements, i.e. types A and B, as shown in Fig. 2(a), are employed. In type A, the FO and UO dendrites interact face-to-face at the converging $\mathrm{GB}$, as in the $2 \mathrm{D}$ case. ${ }^{11)} \mathrm{In}$ type $\mathrm{B}$, the UO dendrite enters between FO dendrites at the converging GB; we refer to this interaction as a space-toface interaction. Here, the four left-most seeds are for the FO grain, the $<100>$ directions of which correspond to the $x$-, $y$-, and $z$-directions, and the five right-most seeds are for the UO grain, the [001] direction of which is inclined at an angle $\theta_{U O}$ measured in the counter-clockwise direction from the $z$-axis. Each seed is placed with a constant spacing of $180 \Delta x$, which is nearly the minimum spacing for two neighboring FO dendrites to grow stably in the $2 \mathrm{D}$ case. The $\mathrm{FO}$ and $\mathrm{UO}$ seeds are referred to as FO1, FO2, ... UO4, and UO5, as shown in Fig. 2(a). In the thin-sample simulations, $n_{x}$ is changed as $n_{x}=1,32,64,128,192$, and 256, whereas $n_{y}$ and $n_{z}$ are constant $\left(n_{y}=n_{z}=1536\right)$. In the large-scale simulations, where multiple FO and UO dendrites interact at the GB, the computational domain size is fixed to $n_{x}=768$ and $n_{y}=n_{z}=1536$, and the initial seed arrangement shown in Fig. 2(b) is employed. For both the thin-sample and the large-scale simulations, the inclination angle $\theta_{U O}$ of the UO grain is changed as $\theta_{U O}=5^{\circ}, 10^{\circ}$, and $15^{\circ}$.

The following parameters were used in the all the simulations: liquidus diffusivity, $D_{l}=3 \times 10^{-9} \mathrm{~m}^{2} / \mathrm{s} ;{ }^{41)}$ solidus diffusivity, $D_{s}=3 \times 10^{-13} \mathrm{~m}^{2} / \mathrm{s}$; ${ }^{41)}$ partition coefficient, $k=$ $0.14 ;{ }^{41)}$ Gibbs-Thomson coefficient, $\Gamma=0.24 \times 10^{-6} \mathrm{~K} \cdot \mathrm{m} ;{ }^{42)}$ liquidus slope, $m=-620 \mathrm{~K} /$ at.frac. $^{43)}$ strength of anisotropy, $\zeta=0.02 ;^{36)}$ interface thickness, $W_{0}=\Delta x / 0.8$; melting temperature of pure $\mathrm{Al}, T_{m}=933.25 \mathrm{~K} ;{ }^{44)}$ pulling velocity, $V_{p}=100 \mu \mathrm{m} / \mathrm{s}$; temperature gradient, $G=10 \mathrm{~K} / \mathrm{mm}$; and time increment, $\Delta t=2.6786 \times 10^{-5} \mathrm{~s}$. Very-long-duration simulations of $5 \times 10^{6}$ and $7 \times 10^{6}$ steps were performed for 


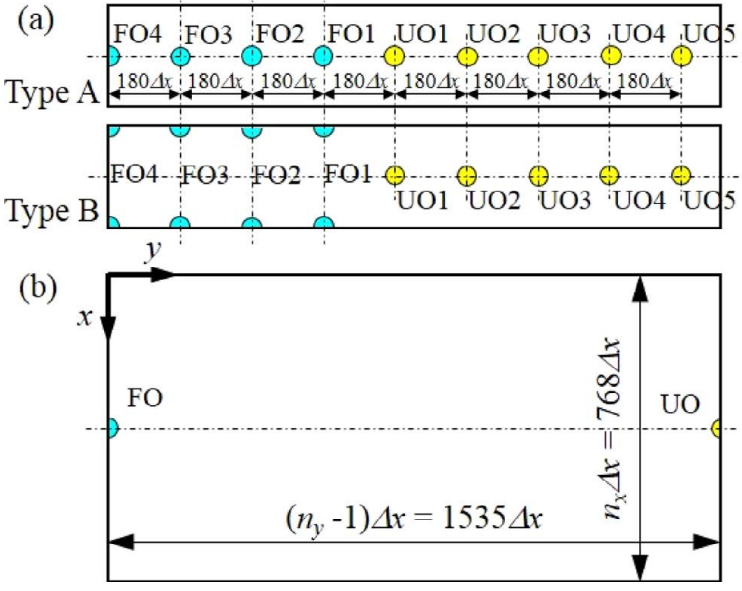

Fig. 2. Initial seed arrangements for (a) thin-sample and (b) largescale simulations. (Online version in color.)

the thin-sample and large-scale simulations, respectively. These correspond to $t=134.0 \mathrm{~s}$ and $187.5 \mathrm{~s}$ and pulling distances of $13.4 \mathrm{~mm}$ and $18.8 \mathrm{~mm}$, respectively. For efficient simulation of directional solidification, the dendrite tip position was kept under $920 \Delta x$ in the $z$-direction until $10^{6}$ steps; after $10^{6}$ steps, one lattice was slid every $\Delta x /\left(V_{\mathrm{p}} \Delta t\right)=$ 280 steps.

\section{Results and Discussion}

\subsection{Thin-sample Simulations}

As an extension of our previous 2D evaluations, ${ }^{11,12)}$ here, we investigated the effects of thin-sample thickness $\left(n_{x}=\right.$ 1 (2D), 32, 64, 128, 192, and 256), UO dendrite inclination angle $\left(\theta_{U O}=5^{\circ}, 10^{\circ}\right.$, and $\left.15^{\circ}\right)$, and dendrite arrangement (types $\mathrm{A}$ and $\mathrm{B}$ ) on the unusual overgrowth behaviors in thin-sample systems with an ideal one-to-one interaction between FO and UO dendrites. Table 1 lists the number of UO dendrites blocked by a FO dendrite. For example, in the case of $\theta_{U O}=5^{\circ}, n_{x}=32$, and type A, we can see that value is " 2 " for the FO1 dendrite. This means that the FO1 dendrite block two UO dendrites (UO1 and UO2) and is overgrown by a third UO dendrite (UO3). All the computations were performed until $5 \times 10^{6}$ steps. Note that "-" in some of the cells implies that the computation was terminated after $5 \times 10^{6}$ steps in these cases. From Table 1, it is observed that the number of UO dendrites required to overgrow an FO dendrite increases with the thin-sample thickness, $n_{x} \Delta x$, and the UO grain inclination angle, $\theta_{U O}$. Regarding the dendrite array type, the difference cannot be determined only from Table 1 . Here, there is no information for $n_{x}=256$ and type $\mathrm{B}$ in Table 1, because the UO dendrites penetrate between the FO dendrite array. Figures 3 and 4 show the dendrite morphologies when $\theta_{U O}=5^{\circ}$ for types $\mathrm{A}$ and $\mathrm{B}$, respectively, just after the FO1 dendrite is overgrown. The upper figures show the side view, and the lower figures show the top view. The solid-liquid interface with $\phi=0$ is shown in light blue for the FO grain and yellow for the UO grain. In the case of $n_{x}=1$ (2D), the solid-liquid interface becomes a line, and the morphologies are identical for both types A and B (see Figs. 3(a) and 4(a)). These figures show that with increasing thin-sample thickness, the growth of secondary arms in the $x$-direction
Table 1. Number of UO dendrites blocked by the FO dendrite. In some cells, "-" implies that the computation was terminated after $5 \times 10^{6}$ steps in these cases.

(a) $\theta_{U O}=5^{\circ}$

\begin{tabular}{cccccc}
\hline$n_{x}$ & type & FO1 & FO2 & FO3 & FO4 \\
\hline 1 & & 1 & 1 & 0 & 0 \\
\hline 32 & A & 2 & 2 & 0 & - \\
& B & 2 & 2 & 0 & - \\
\hline \multirow{2}{*}{64} & A & 3 & 1 & $1-$ & \\
& B & 3 & 1 & $1-$ & \\
\hline 128 & A & 3 & 1 & $1-$ & \\
\hline 192 & B & 3 & 1 & $1-$ & \\
\hline 256 & A & 3 & 2 & - & \\
\hline
\end{tabular}

(b) $\theta_{U O}=10^{\circ}$

\begin{tabular}{cccccc}
\hline$n_{x}$ & type & FO1 & FO2 & FO3 & FO4 \\
\hline 1 & & 1 & 4 & 0 & 0 \\
\hline 32 & A & 4 & 1 & 3 & 0 \\
& B & 4 & 1 & 2 & 1 \\
\hline 64 & A & 6 & $4-$ & & \\
& B & 5 & $6-$ & & \\
\hline 128 & A & $12-$ & & & \\
\hline 192 & B & $12-$ & & & \\
\hline 256 & A & $12-$ & & & \\
\hline
\end{tabular}

(c) $\theta_{U O}=15^{\circ}$

\begin{tabular}{ccc}
\hline$n_{x}$ & type & FO1 \\
\hline 1 & & 3 \\
\hline 32 & A & $14-$ \\
& B & $15-$ \\
\hline 64 & A & $16-$ \\
& B & $15-$ \\
\hline \multirow{2}{*}{128} & A & $16-$ \\
& B & $16-$ \\
\hline \multirow{2}{*}{192} & A & $18-$ \\
& B & $17-$ \\
\hline 26 & A & $18-$ \\
\hline
\end{tabular}

(thickness direction) becomes more remarkable. Figure 5 shows the tip trajectories of the FO1 dendrite when $\theta_{U O}=$ $5^{\circ}$. The blue and red lines represent types A and B, respectively. The dashed line represents the original seed position of the FO1 dendrite. The tip trajectories are drawn until the tip position reaches the 870th lattice point in the $z$-direction. The ends of the tip trajectories except for $n_{x}=256$ of type B correspond to Figs. 3 and 4. Figure 6 shows time slices of the top views during interactions between the FO1 and UO3 dendrites at the converging GB for $n_{x}=192, \theta_{U O}=$ $5^{\circ}$, and type A. Here, the dendrite surface $(\phi=-0.9)$ and 

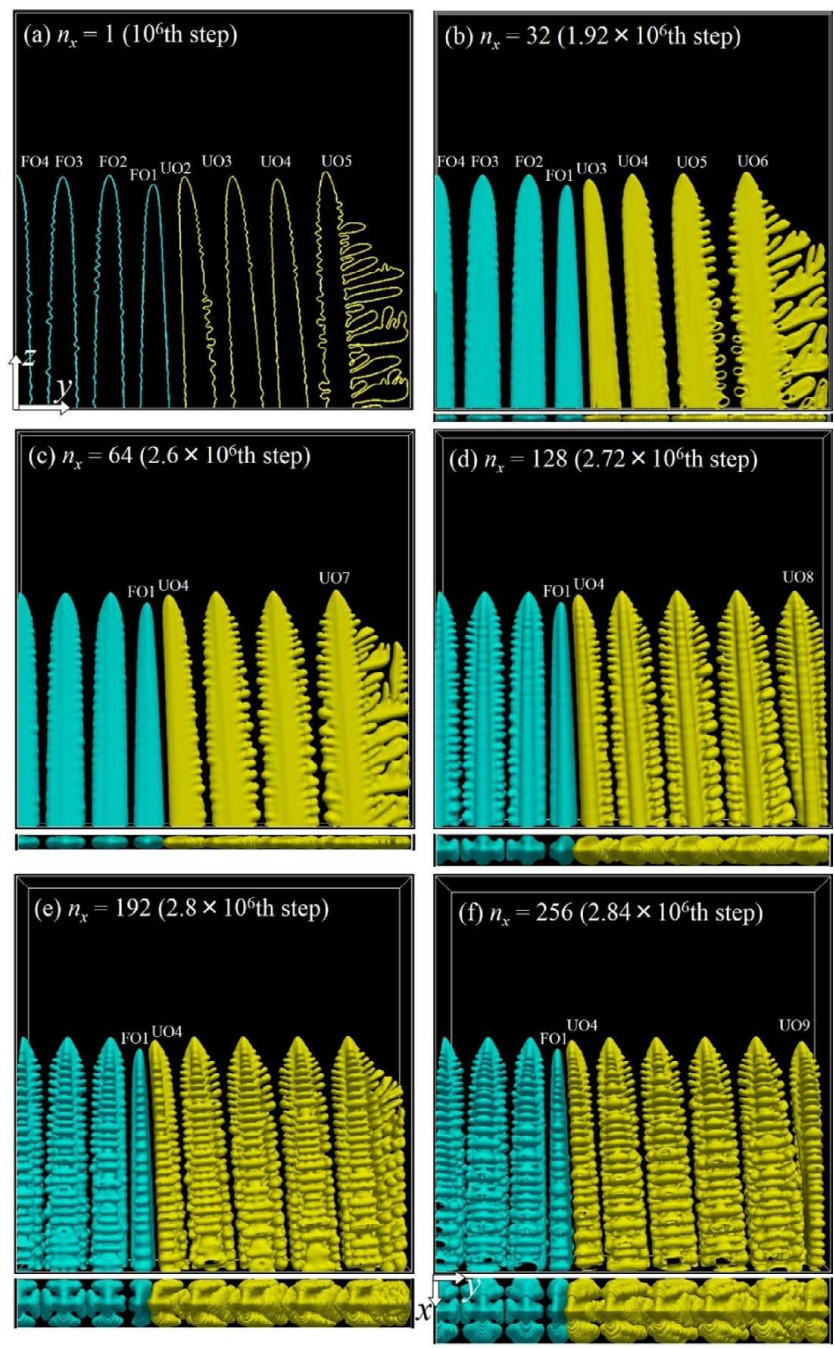

Fig. 3. Dendrite morphologies in thin-sample simulations for type A with $\theta_{U O}=5^{\circ}$. The upper figures show the side view from the positive $x$-axis direction, and the bottom figures show the top view from the positive $z$-axis direction. The solid-liquid interface with $\phi=0$ is shown in light blue for the FO grain and yellow for the UO grain. Each time step is nearly immediately after overgrowth of the FO1 dendrite. (Online version in color.)

concentration distribution on the surface at the 880th lattice point in the $z$-direction are illustrated. Figures 6(a) to 6(e) correspond to the points indicated by "a" to "e" in Fig. 5(d). Two dashed lines indicate the tip positions of FO1 dendrite at $1.54 \times 10^{6}$ th step, just after the UO2 dendrite is overgrown, and at $2.24 \times 10^{6}$ th step, just after the UO3 dendrite is overgrown. In Fig. 6(a), each solute layer around a dendrite appears isolated and the solute layer interactions are small. The interaction of solute layers around the FO1 and UO3 dendrites increases with time as shown in Figs. 6(b), 6(c), and 6(d). Then, the surface areas of the FO1 and UO3 dendrites are decreased with time. This indicates the falling of these dendrite tips, as shown for the FO1 dendrite in Fig. 5(d). Here, the falling amount is larger for the UO3 dendrite than that for FO1, and the UO3 dendrite is finally overgrown as shown in Fig. 6(e). The FO1 dendrite tip position is moved to the left. After this, the FO1 dendrite is overgrown by the UO4 dendrite, because the spacing between the FO1 and FO2 dendrites becomes smaller than the critical distance. From Fig. 6, it is clearly seen that the
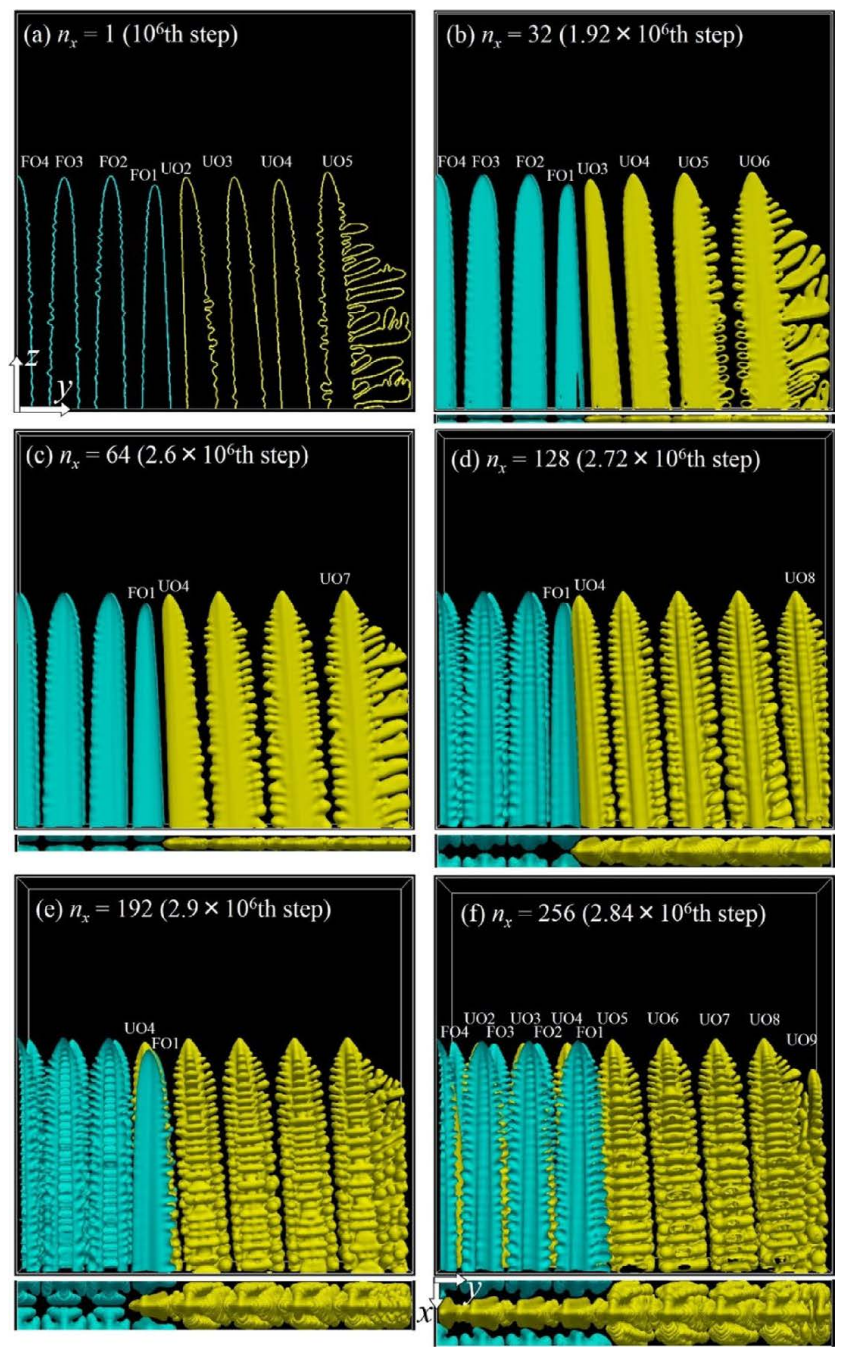

Fig. 4. Dendrite morphologies in thin-sample simulations for type B with $\theta_{U O}=5^{\circ}$. The upper figures show the side view from the positive $x$-axis direction, and the bottom figures show the top view from the positive $z$-axis direction. The solid-liquid interface with $\phi=0$ is shown in light blue for the FO grain and yellow for the UO grain. Each time step is nearly immediately after overgrowth of the FO1 dendrite. (Online version in color.)

unusual overgrowth occurs because of the solute interaction around the dendrite tips.

The effect of UO dendrite inclination angle, $\theta_{U O}$, on the unusual overgrowth is similar to that in the $2 \mathrm{D}$ case. ${ }^{11)}$ As clearly seen in Table 1, the number of UO dendrites required to overgrow the FO1 dendrite increases with $\theta_{U O}$. The tip lag of the UO dendrite as compared to the FO dendrite increases with $\theta_{U O}{ }^{3)}$ Then, as the UO dendrite approaches the FO dendrite at the converging GB, the horizontal migration of the FO dendrite decreases as $\theta_{U O}$ increases. In addition, because the migration rate of the UO dendrite with large inclination is higher than that with small inclination, the interaction period between FO and UO dendrites at the converging GB decreases. These are the reasons why the unusual overgrowth needs more time when $\theta_{U O}$ becomes large. ${ }^{11,12)}$ In the case of $\theta_{U O}=15^{\circ}$, the FO1 dendrites were not overgrown until $5 \times 10^{6}$ steps except when $n_{x}=1$. However, when the UO dendrites approached the FO1 dendrite, the FO1 dendrite migrated to the FO2 dendrite side in all the cases. This means that, if the simulation is prolonged, 


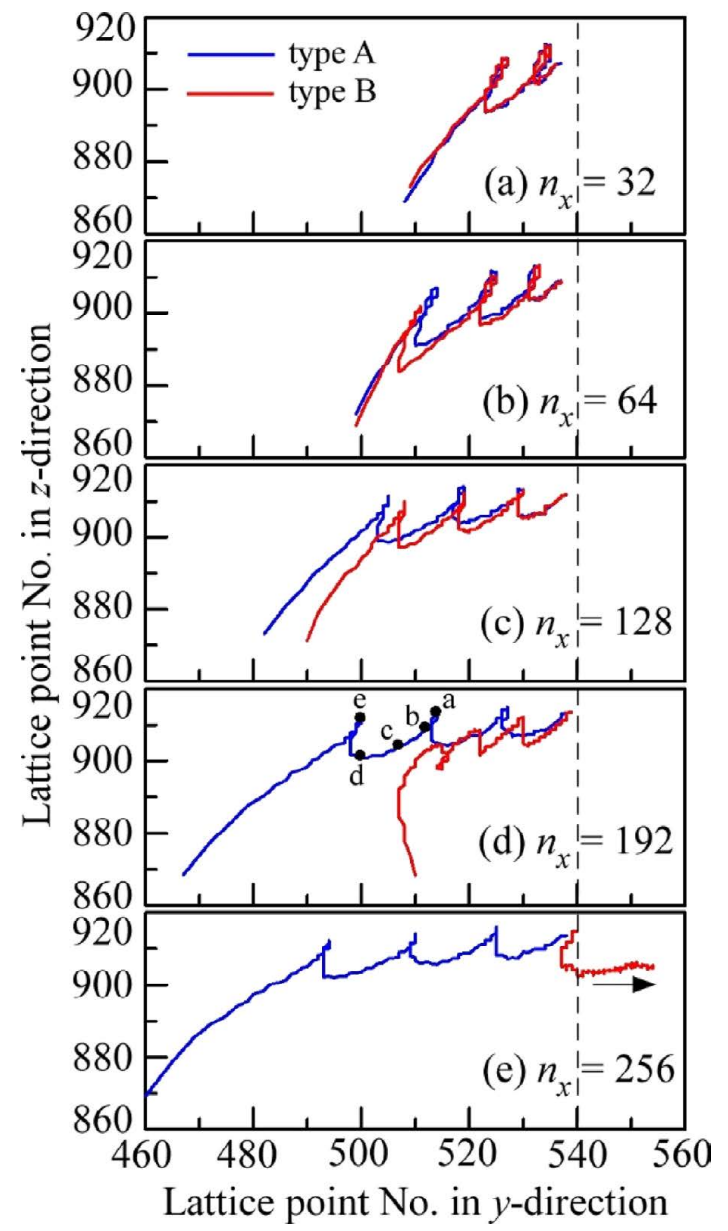

Fig. 5. Tip trajectories of FO1 dendrite from the $0.24 \times 10^{6}$ th step until when the FO1 dendrite is overgrown or the $5 \times 10^{6}$ th step only for type B with $n_{x}=256$. (Online version in color.) all the FO dendrites will be overgrown.

The interaction between FO and UO dendrites at the converging GB occurs because of the overlap of solute layers. ${ }^{11)}$ In the $3 \mathrm{D}$ case, the solute rejected into the liquid phase from the solid phase can diffuse in any direction, in contrast to the constrained 2D case. Hence, the interaction between FO and UO dendrites at the converging GB decreases as the sample thickness increases. This can be seen in Fig. 3, where the spacing between FO1 and the UO dendrite at the converging $\mathrm{GB}$ is smaller for larger sample thickness. Moreover, Fig. 3 shows that the FO1 dendrite position when the FO1 dendrite is just overgrown lies more toward the left side as the sample thickness increases. This is because the minimum dendrite spacing at which two neighboring FO dendrites can keep growing decreases as the sample thickness increases. In Fig. 5, consider only the type A lines. When an UO dendrite approaches the FO1 dendrite, the FO1 dendrite migrates to the left and falls. Then, when the UO dendrite is overgrown by the FO1 dendrite, the tip position of the FO1 dendrite rises again. In the case of small sample thickness, the tip migrates slightly to the right. However, the amount of horizontal migration to the right decreases with increasing sample thickness. On the other hand, the amount of horizontal migration to the left when an UO dendrite approaches the FO1 dendrite increases with the sample thickness. This is because the minimum spacing of the FO dendrites at which two neighboring FO dendrites can keep growing decreases with increasing sample thickness. This can also be seen in the case of the UO dendrites. In Fig. 3, the number of UO dendrite increases from four for $n_{x}=1$ to six for $n_{x}=256$. In addition, from Table $1(\mathrm{c})$, when $\theta_{U O}=15^{\circ}$, it can be seen that the number of UO dendrites blocked until $5 \times 10^{6}$ steps increases with $n_{x}$. This is because the spacing of the UO
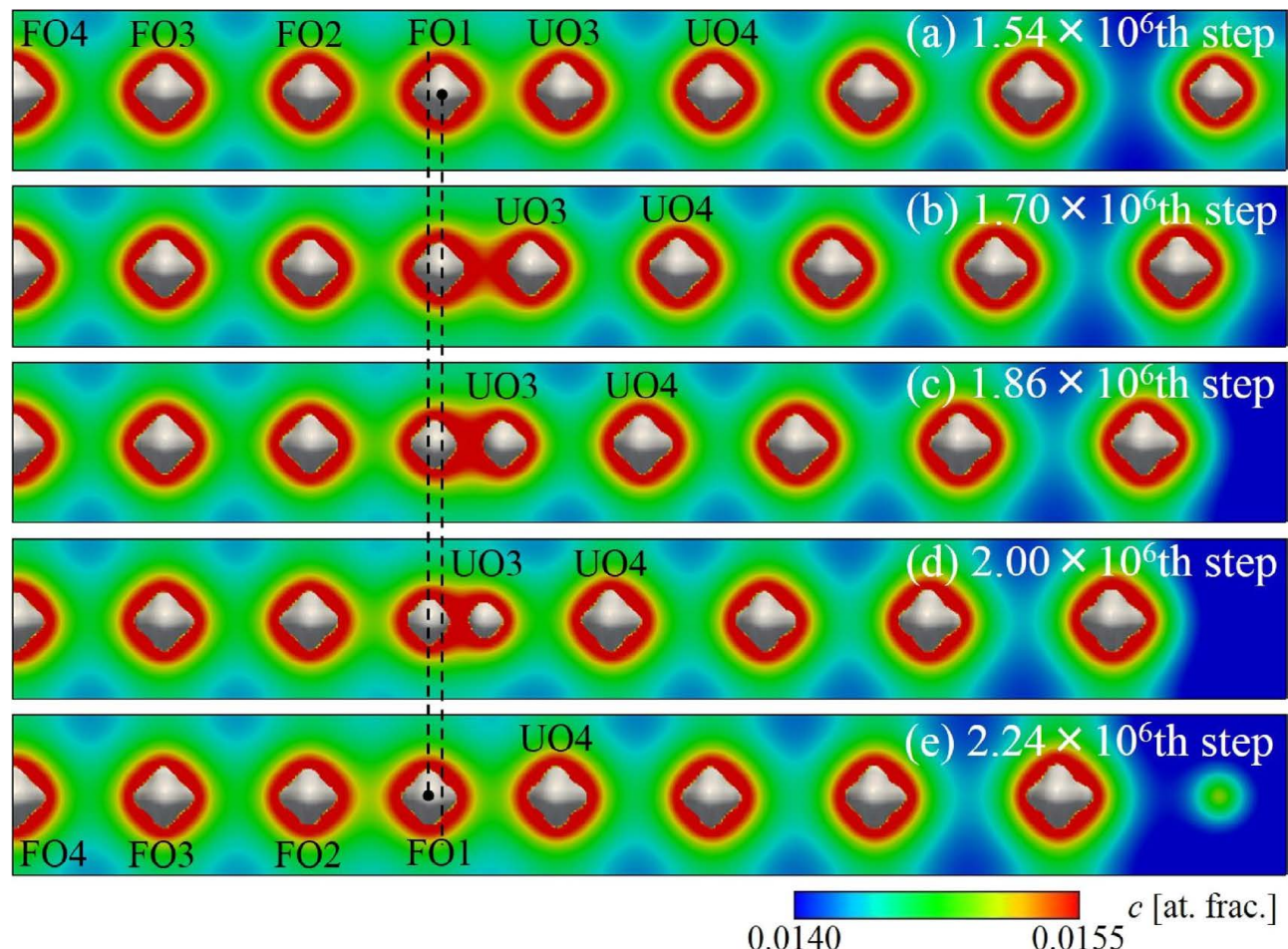

Fig. 6. Time slices of top views of dendrite surface $(\phi=-0.9)$ and concentration distribution on the surface at the 880th lattice point in the $z$-direction for $n_{x}=192, \theta_{U O}=5^{\circ}$, and type A: (a) $1.54 \times 10^{6}$ th step (41.3 s), (b) $1.70 \times 10^{6}$ th step $(45.5 \mathrm{~s})$, (c) $1.86 \times 10^{6}$ th step $(49.8 \mathrm{~s})$, (d) $2.00 \times 10^{6}$ th step $(53.6 \mathrm{~s})$, and (e) $2.24 \times 10^{6}$ th step $(60.0 \mathrm{~s})$. (Online version in color.) 
dendrites decreases with increasing thin-sample thickness. As a result, the unusual overgrowth becomes more difficult with increasing sample thickness.

Comparing types A and B in Figs. 3 and 4, for $n_{x}=32$ and 64, we can see nearly the same morphologies. However, when $n_{x}=128$ and 192 for type B, we can see an overlap of FO1 and UO4 dendrites in the side view. From the top views, it can be observed that the UO4 dendrites penetrate into the FO1 dendrite spacing. Figure 7 shows the tip trajectories of the FO1 and UO1-4 dendrites when $n_{x}=192$ for types A and B. Note that the tip trajectories of UO1-3 are considerably different for types $\mathrm{A}$ and $\mathrm{B}$, where the UO dendrites of type B can migrate more toward the left. The tip trajectories of the FO1 dendrite are also different for both types. Because the interaction in the $y$-direction is larger for type A, i.e., face-to-face interaction, the FO1 dendrite of type A migrates largely to the left. However, interestingly, the FO1 dendrites for types A and B are overgrown

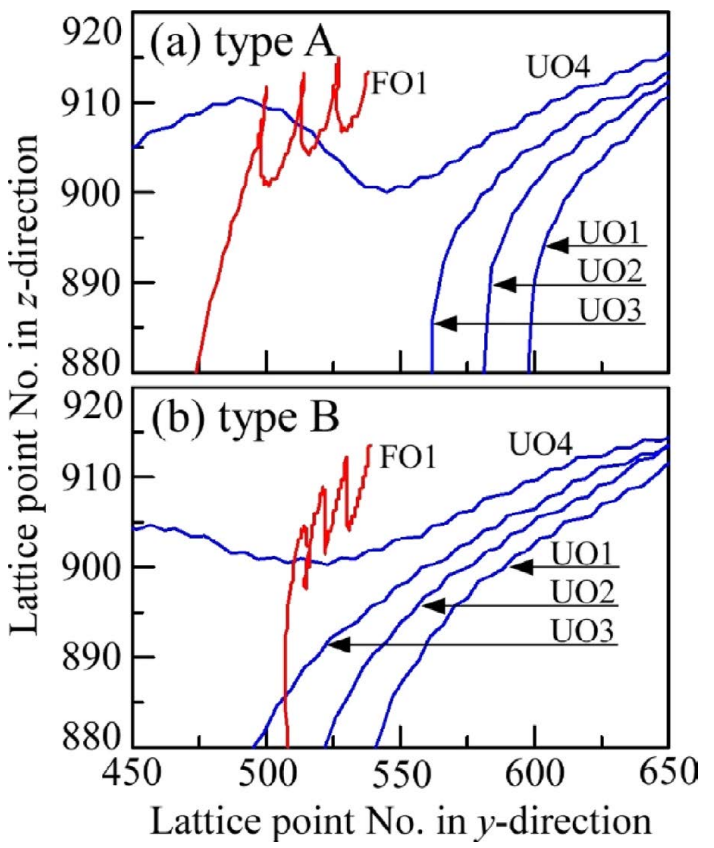

Fig. 7. Tip trajectories of FO1 and UO1-4 for (a) type A and (b) type B when $n_{x}=192$. (Online version in color.) by the same UO4 dendrite. This is due to the interaction in the thickness direction for type $\mathrm{B}$, i.e., space-to-face interaction. Moreover, from Fig. 4(f), i.e., when $n_{x}=256$, we can see that the UO dendrites penetrate between the FO dendrites. In this case, as shown in Fig. 5(e), the FO1 dendrite migrates to the right while its tip position is nearly constant in the $z$-direction. Figure $\mathbf{8}$ shows the top views for $n_{x}=256, \theta_{U O}=5^{\circ}$, and type B at the (a) $3 \times 10^{6}$ th step and (b) $3.37 \times 10^{6}$ th step. Here, the dendrites are expressed by a contour surface with $\phi=-0.9$ and the solute concentration distributions on the $x-y$ surface at the 800th lattice point in the $z$-direction. In Fig. 8(a), there is a relatively low concentration area to the right of the FO1 dendrite (indicated by thick arrows). Then, as shown in Fig. 8(b), the FO1 dendrite can move to the right. Thus, the unusual overgrowth never occurs for $n_{x}=256$ and type B. It can be concluded that, as clearly seen in Fig. 5, the interaction between FO and UO dendrites differs in the face-to-face and space-to-face cases when the thin-sample thickness is relatively large.

\subsection{Large-scale Simulations for Multiple Dendrites Interaction}

In the previous thin-sample simulations with an ideal one-to-one interaction between FO and UO dendrites at the converging GB, we found that, in the case of small thickness, the interaction between FO and UO dendrites at the converging GB is nearly the same for face-to-face and space-to-face interactions; on the other hand, as the thickness increases, the interaction gradually changes depending on the dendrite array, and the penetration of UO dendrites between FO dendrites occurs for the space-to-face interaction when the thin-sample thickness achieve a critical value. Here, we investigate what happens in a realistic multiple dendrite interaction system that includes both of face-toface and space-to-face interactions. In the computational condition shown in Figs. 1 and 2(b), we used $n_{x} \times n_{y} \times n_{z}=$ $768 \times 1536 \times 1536$ lattice points with the lattice size $\Delta x=$ $0.75 \mu \mathrm{m}$, and performed very-long-duration simulations with $7 \times 10^{6}$ steps $(t=187.5 \mathrm{~s})$. Three simulations were performed for $\theta_{U O}=5^{\circ}, 10^{\circ}$, and $15^{\circ}$. In each simulation, a 386 GPU was used, and the computational time was around 5.5 days.

(a) $3 \times 10^{6}$ th step

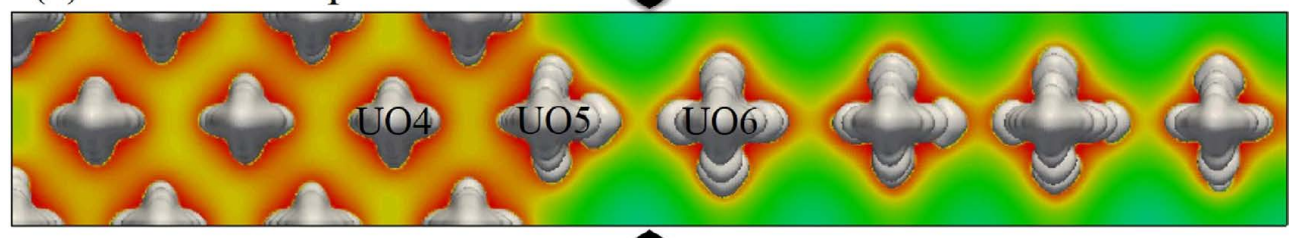

(b) $3.37 \times 10^{6}$ th step

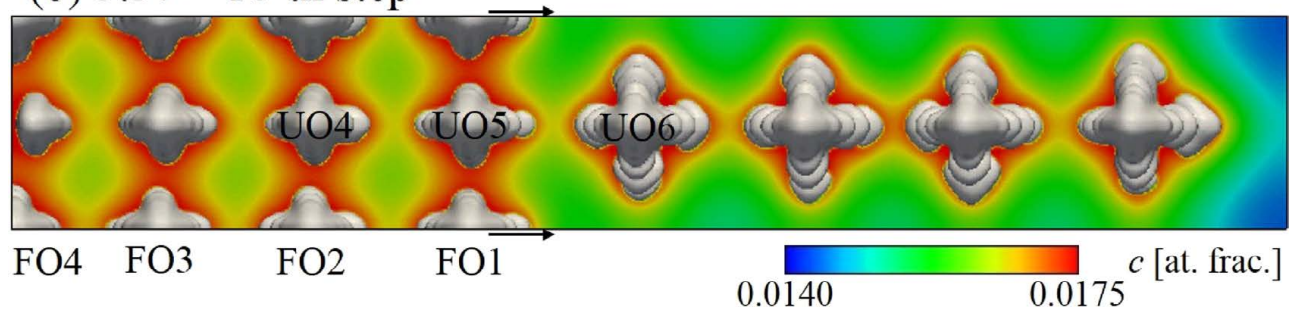

Fig. 8. Top views of dendrite surface $(\phi=-0.9)$ and concentration distribution on the surface at the 800th lattice point in the $z$-direction for $n_{x}=256, \theta_{U O}=5^{\circ}$, and type B. (Online version in color.) 

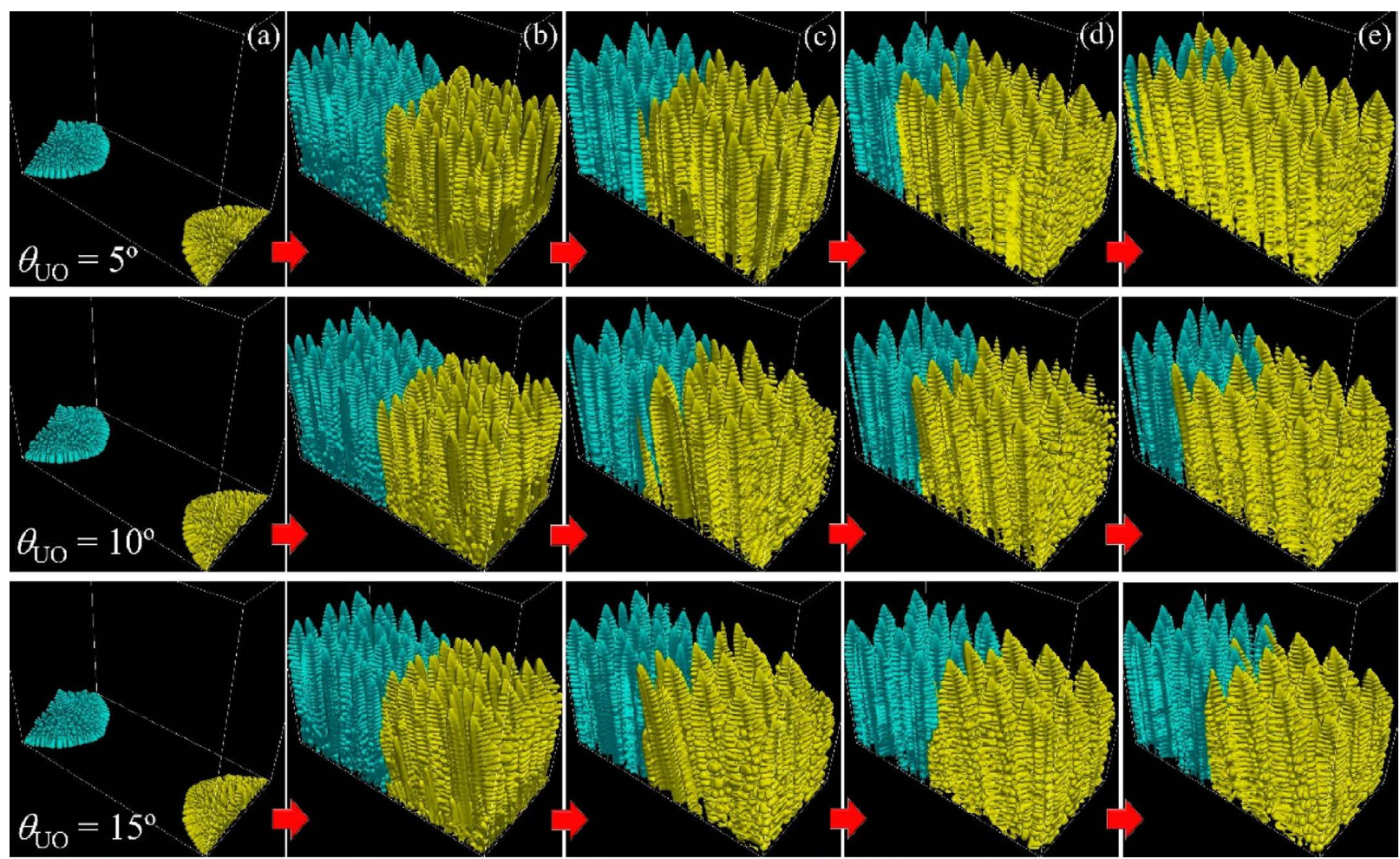

Fig. 9. Time slices of large-scale simulations with $n_{x} \times n_{y} \times n_{z}=768 \times 1536 \times 1536$ : (a) $0.05 \times 10^{6}$ th step (1.3 s), (b) $0.25 \times 10^{6}$ th step $(6.7 \mathrm{~s})$, (c) $10^{6}$ th step $(26.8 \mathrm{~s})$, (d) $3 \times 10^{6}$ th step $(80.4 \mathrm{~s})$, and (e) $7 \times 10^{6}$ th step $(187.5 \mathrm{~s})$. (Online version in color.)

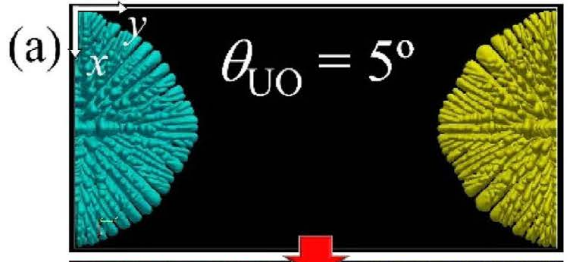

(b)

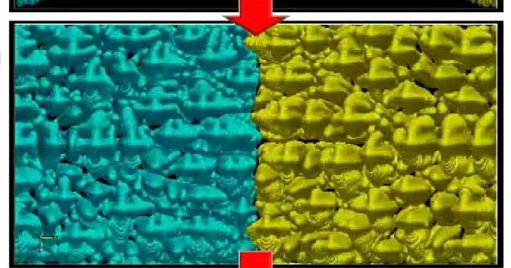

(c)

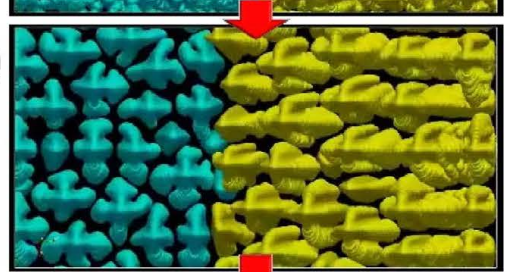

(d)
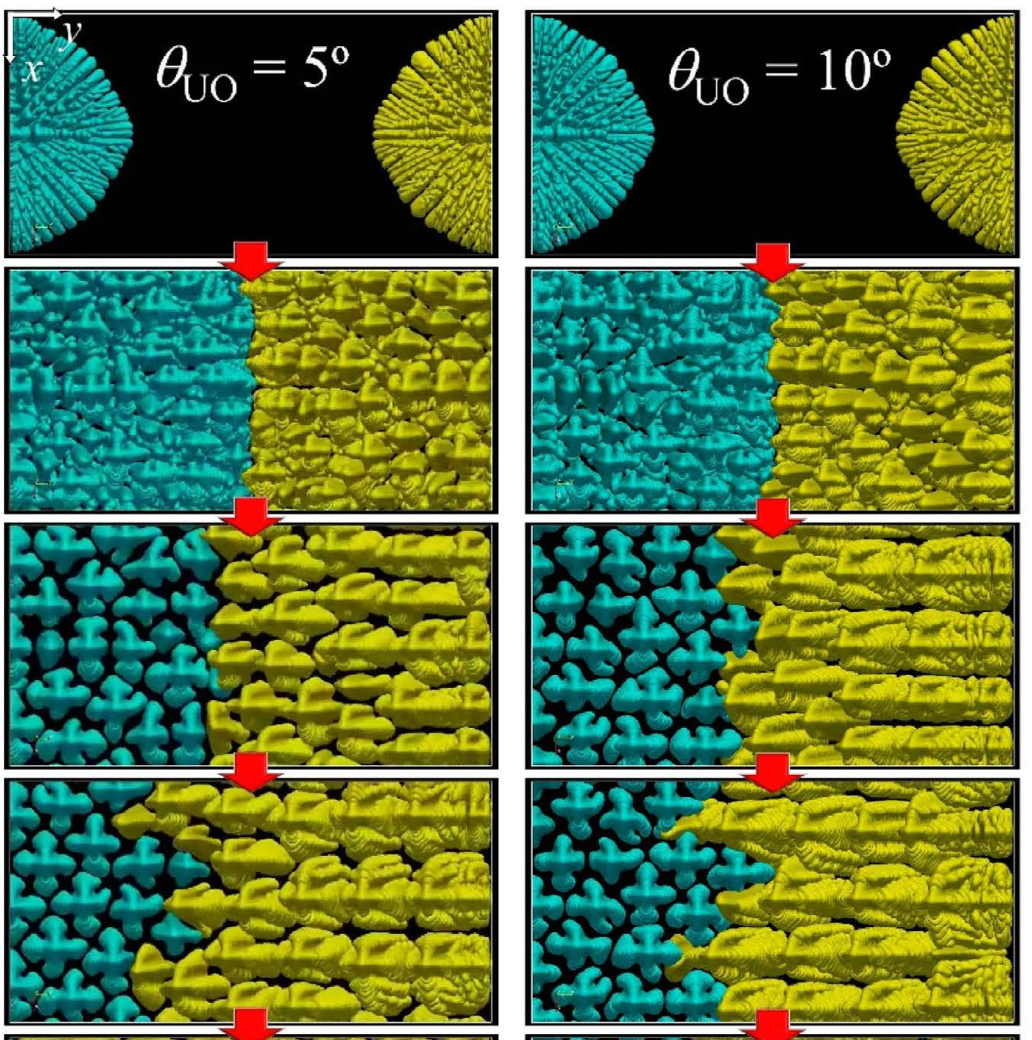

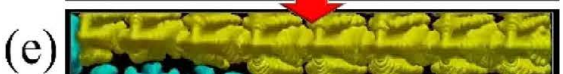
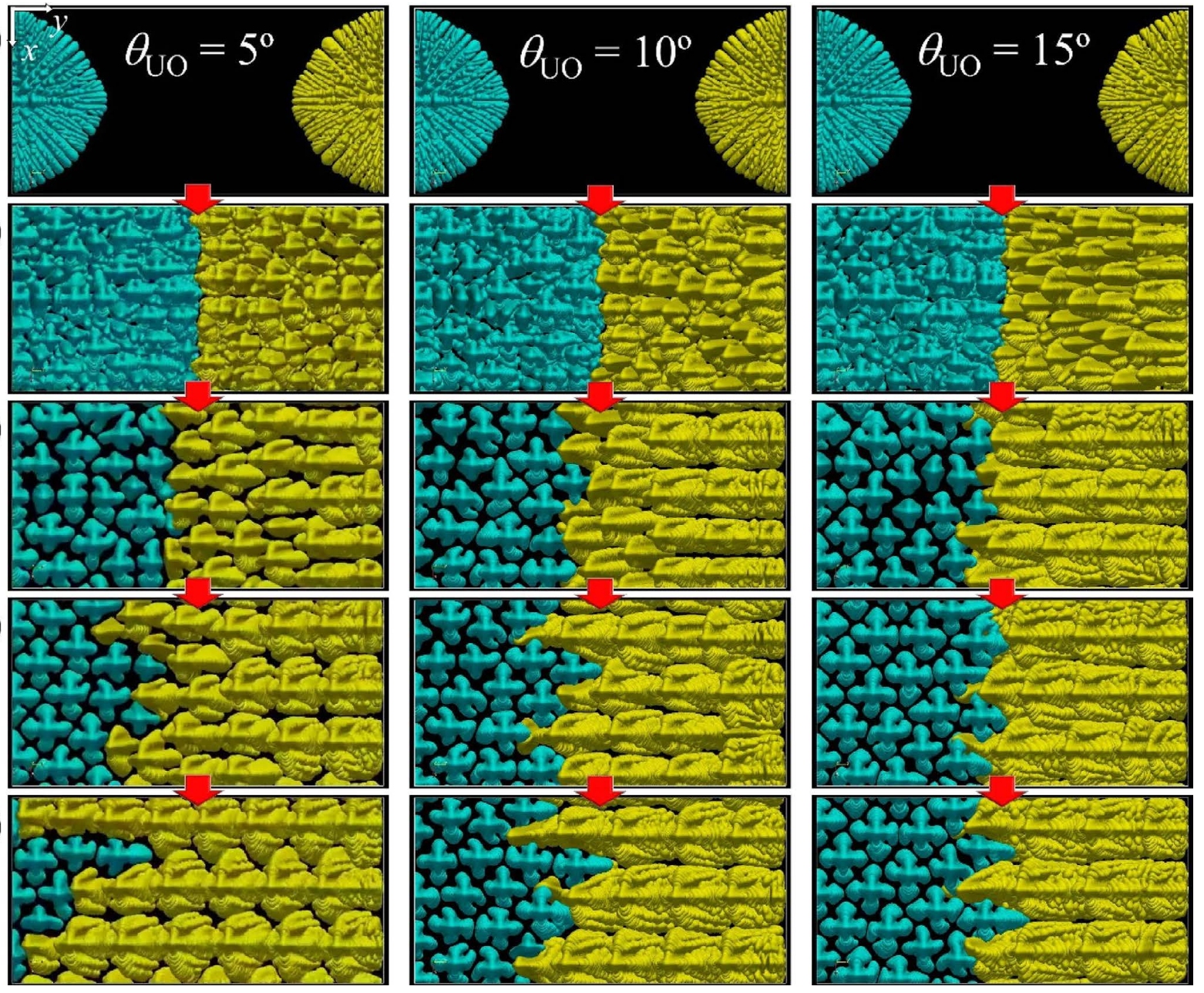

Fig. 10. Top views of Fig. 8: (a) $0.05 \times 10^{6}$ th step (1.3 s), (b) $0.25 \times 10^{6}$ th step $(6.7 \mathrm{~s})$, (c) $10^{6}$ th step $(26.8 \mathrm{~s})$, (d) $3 \times 10^{6}$ th step ( $80.4 \mathrm{~s})$, and (e) $7 \times 10^{6}$ th step (187.5 s). (Online version in color.) 
Figures 9 (bird's-eye view) and $\mathbf{1 0}$ (top view) show the time slices at the (a) $0.05 \times 10^{6}$ th step $\left(1.3 \mathrm{~s}\right.$ ), (b) $0.25 \times 10^{6}$ th step $(6.7 \mathrm{~s})$, (c) $10^{6}$ th step $(26.8 \mathrm{~s})$, (d) $3 \times 10^{6}$ th step $(80.4 \mathrm{~s})$, and (e) $7 \times 10^{6}$ th step $(187.5 \mathrm{~s})$. As in Figs. 3 and 4 , the solid-liquid interface surface with $\phi=0$ is shown in light blue for the FO grain and yellow for the UO grain. First, the solid wets the bottom surface (Figs. 9(a) and 10(a)). Then, two grains collide at nearly the center of the domain, and form a straight GB (Fig. 10(b)). Here, we can see many dendrites arranged randomly, and the inclination angle of the UO grain is smaller than $\theta_{U O}$ (Fig. 9(b)). As the simulations proceed, selection of dendrites in the FO and UO grains occurs, and the dendrite arrangements are gradually ordered. In the FO grain, a hexagonal arrangement can be observed from around the $10^{6}$ th step (Figs. 9(c) and $10(\mathrm{c})$ ). In the UO grain, the dendrites are gradually ordered in both the migration direction (y-direction) and the lateral direction ( $x$-direction). Finally, the UO dendrites are arranged along three lines (Fig. 10(e)) for all $\theta_{U O}$. During the coarsening of the UO dendrites, the inclination increases as shown in Figs. 9(b)-9(e). For all $\theta_{U O}$, unusual overgrowth is observed, where the UO dendrites penetrate more deeply into the FO grain as $\theta_{U O}$ decreases. Interestingly, the unusual overgrowth rate differs at the GB,

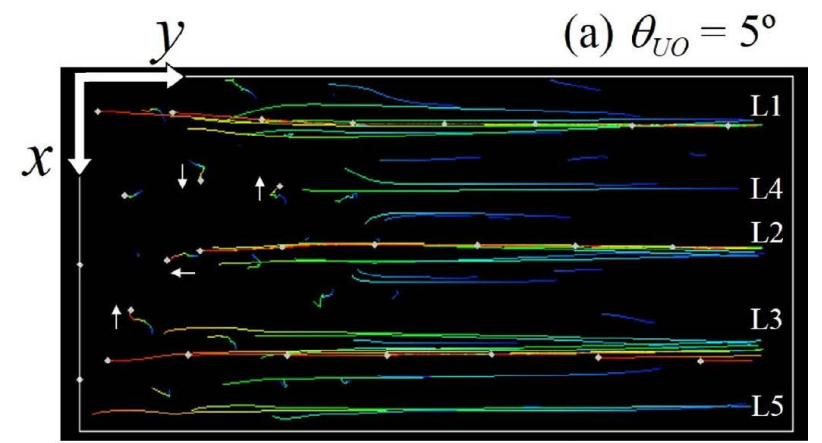

(b) $\theta_{U O}=10^{\circ}$

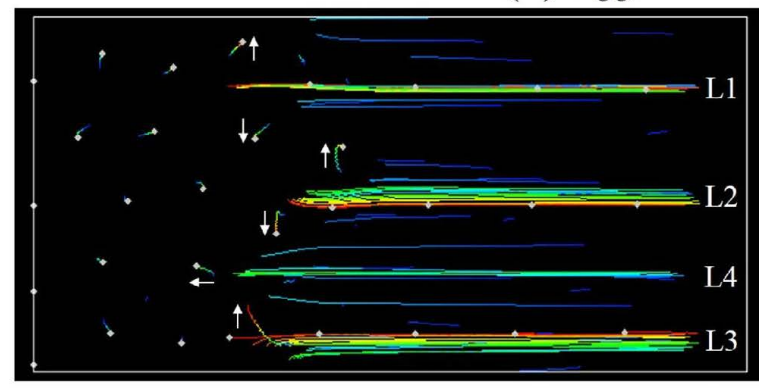

(c) $\theta_{U O}=15^{\circ}$

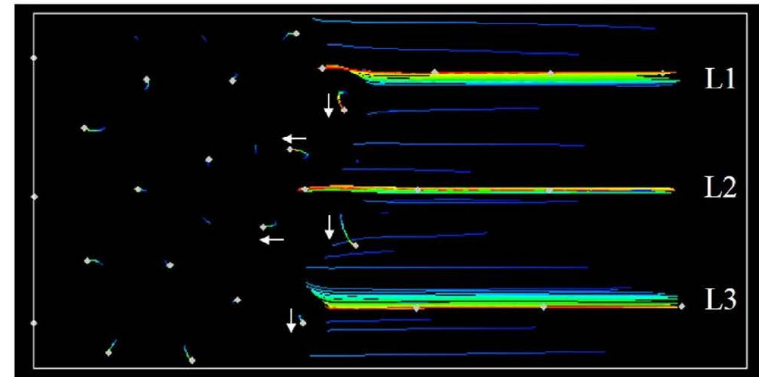

Fig. 11. Top views of tip trajectories in the large-scale simulations. The colors indicate time from the $0.22 \times 10^{6}$ th step (blue) to the $7 \times 10^{6}$ th step (red). The white marks denote the tip positions in the final $\left(7 \times 10^{6}\right.$ th) steps. (Online version in color.) i.e., the GB becomes zigzag or inlet-like in shape. In Fig. $10(\mathrm{e})$, the depth of the zigzag GB is deeper for smaller $\theta_{U O}$. When $\theta_{U O}=10^{\circ}$ and $15^{\circ}$, some secondary arms of the FO dendrites at the GB overhang toward the UO grain side and reach toward the right as compared to the position of the initial GB shown in Fig. 10(b).

Figure 11 shows the tip trajectories of FO and UO dendrites from the $0.22 \times 10^{6}$ th step to the $7 \times 10^{6}$ th step of the large-scale simulations shown in Figs. 9 and 10. The line color indicates the time from blue $\left(0.22 \times 10^{6}\right.$ th step $)$ to red $\left(7 \times 10^{6}\right.$ th step $)$. The white marks are the tip positions in the final, i.e., $7 \times 10^{6}$ th step; the lines without white mark at their ends denote overgrowth. In three simulations with different $\theta_{U O}$, three UO dendrite lines are finally formed, as shown in Fig. 10(e). Hence, in Fig. 11, these three lines are referred to as L1-L3. Lines L4 and L5 shown in Figs. 11(a) and 11(b) are the UO dendrite lines that eventually disappear. In addition, the migration directions of some FO dendrites at the GB are indicated by white arrows. The overgrowth of lines L4 and L5 occurred by the branching of UO dendrites on lines L1-L3 at the right end. This can be seen in Fig. 9(c) for L4 and Fig. 10(d) for L5 when $\theta_{U O}=5^{\circ}$, and Fig. 9(d) for L4 when $\theta_{U O}=10^{\circ}$. For $\theta_{U O}=5^{\circ}$, when selecting L4 and L5, L2 and L3 migrate upward and downward, respectively, to adjust the spacing between the UO dendrites in the lateral direction. For $\theta_{U O}=10^{\circ}$, when selecting L4, L2 and L3 migrate downward and upward, respectively. We can also see the lateral migration of the UO dendrite lines for $\theta_{U O}=15^{\circ}$. Furthermore, it can be observed that, when $\theta_{U O}=5^{\circ}$, the UO dendrite lines L1-L3 are slightly curved so as to avoid the FO dendrites. As $\theta_{U O}$ increases, the UO dendrite lines become straight. This is because the migration rate of the UO dendrite increases with $\theta_{U O}$, and the interaction period with the FO dendrite becomes shorter. Meanwhile, we can see a slight curve of the tip trajectories of the UO dendrites at around termination, because the UO dendrites try to enter between the FO dendrites. The FO dendrites at the GB indicated by arrows migrate to avoid the UO dendrites. The curve of UO dendrite lines for avoiding the FO dendrites and the lateral migrations of the FO dendrite to avoid the UO dendrites indicate a rearrangement of FO and UO dendrites toward the space-to-face interaction. The perfect face-to-face interaction is thought to be rare in the real multiple dendrite interaction system, and there is a certain degree of misalignment between FO and UO dendrites. Then, such a rearrangement will occur and the UO dendrites penetrate into the FO grain. We can see the face-to-face interaction in front of the L2 line in Fig. 11(a), and the unusual overgrowth is delayed there. This interaction difference at the GB forms the zigzag GB.

\section{Conclusions}

Phase-field studies of 3D dendrite competitive growth at the converging GB of a bicrystal binary alloy were performed. First, a series of thin-sample simulations were conducted to investigate the effects of sample thickness, UO grain inclination angle, and dendrite arrangement on the dendrite competitive growth. Next, realistic large-scale simulations were conducted, whereby multiple dendrites interact at the converging GB. The simulations were per- 
formed via parallel GPU computation using the TSUBAME 2.5 supercomputer at Tokyo Institute of Technology. The following conclusions were obtained:

(1) Unusual overgrowth occurs not only in the thin sample but also in the bulk material; this was reproduced by the large-scale 3D phase-field simulations.

(2) Unusual overgrowth easily occurs when the sample thickness and the inclination angle of the UO dendrite are small.

(3) In the case of a very thin sample, the interaction between FO and UO dendrites at the converging GB is independent of the dendrite arrangement. On the other hand, when the thickness of the thin sample increases, the interaction depends on the dendrite arrangement. In the case of space-to-face interaction, if the space exceeds a critical value, the UO dendrites penetrate between the FO dendrites.

(4) In the large-scale simulations with multiple dendrite interaction, a zigzag GB was observed, and the depth of the zigzag pattern was larger for UO grains with a smaller inclination angle.

(5) The FO and UO dendrites were rearranged toward a space-to-face interaction.

Our large-scale parallel GPU computation facilitated detailed investigations of the realistic $3 \mathrm{D}$ dendrite interactions at the converging GB. To the best of our knowledge, this is the first such instance. Although we treated a set of crystal orientations for FO and UO grains in this study, there are many other combinations of crystal orientations in the $3 \mathrm{D}$ case. We expect that various competitive growth phenomena between grains will be clarified by performing systematical large-scale phase-field simulations.

\section{Acknowledgements}

This research was supported by the 22th ISIJ Research Promotion Grants from the Iron and Steel Institute of Japan (ISIJ), Grant-in-Aid for Scientific Research (B) (No. 25289006 and No. 25289266) from Japan Society for the Promotion of Science (JSPS), Grant-in-Aid for Scientific Research (S) (No. 26220002) from the Ministry of Education, Culture, Sports, Science and Technology (MEXT), Joint Usage/Research Center for Interdisciplinary Largescale Information Infrastructures (JHPCN) and High Performance Computing Infrastructure (HPCI) in Japan, and the Computational Materials Science Initiative (CMSI). The authors thank the Global Scientific Information and Computing Center, Tokyo Institute of Technology for allowing the use of the TSUBAME 2.5 supercomputer.

\section{REFERENCES}

1) T. F. Bower and M. C. Flemings: Trans. AIME, 239 (1967), 1620.
2) D. Walton and B. Chalmers: Trans. Metall. AIME, 215 (1959), 447.

3) M. Rappaz and C. A. Gandin: Acta Metall. Mater., 41 (1993), 345.

4) C. A. Gandin and M. Rappaz: Acta Metall. Mater., 42 (1994), 2233.

5) H. Esaka, M. Tamura and K. Shinozuka: Mater. Trans., 44 (2003), 829.

6) N. D’Souza, M. G. Ardakani, A. Wagner, B. A. Shollock and M. McLean: J. Mater. Sci., 37 (2002), 481.

7) A. Wagner, B. A. Shollock and M. McLean: Mater. Sci. Eng., A, 374 (2004), 270.

8) Y. Z. Zhou, A. Volek and N. R. Green: Acta Mater., 56 (2008), 2631.

9) H. Yu, J. Li, X. Lin, L. Wang and W. Huang: J. Cryst. Growth, 402 (2014), 210.

10) J. Li, Z. Wang, Y. Wang and J. Wang: Acta Mater., 60 (2012), 1478.

11) T. Takaki, M. Ohno, T. Shimokawabe and T. Aoki: Acta Mater., 81 (2014), 272

12) Y. Shibuta, M. Ohno and T. Takaki: JOM, 67 (2015), 1793.

13) D. Tourret and A. Karma: Acta Mater., 82 (2015), 64

14) T. Takaki, M. Ohno, Y. Shibuta, S. Sakane, T. Shimokawabe and T. Aoki: J. Cryst. Growth, 442 (2016), 14.

15) T. Takaki: ISIJ Int., 54 (2014), 437.

16) T. Takaki, T. Shimokawabe, M. Ohno, A. Yamanaka and T. Aoki: $J$. Cryst. Growth, 382 (2013), 21.

17) X. B. Meng, Q. Lu, X. L. Zhang, J. G. Li, Z. Q. Chen, Y. H. Wang, Y. Z. Zhou, T. Jin, X. F. Sun and Z. Q. Hu: Acta Mater., 60 (2012), 3965 .

18) Z. Liu, M. Lin, D. Yu, X. Zhou, Y. Gu and H. Fu: Metall. Mater. Trans. A, 44 (2013), 5113.

19) C. Yang, L. Liu, X. Zhao, N. Wang, J. Zhang and H. Fu: J. Alloys Compd., 578 (2013), 577.

20) P. Carter, D. C. Cox, C. A. Gandin and R. C. Reed: Mater. Sci. Eng. A, 280 (2000), 233.

21) D. Pan, Q. Xu, B. Liu, J. Li, H. Yuan and H. Jin: JOM, 62 (2010), 30 .

22) H. J. Dai, N. D'Souza and H. B. Dong: Metall. Mater. Trans. A, 42 (2011), 3430.

23) R. Kobayashi: Physica D, 63 (1993), 410.

24) I. Steinbach: JOM, 65 (2013), 1096

25) A. Karma and D. Tourret: Curr. Opin. Solid State Mater. Sci., 20 (2016), 25.

26) A. Karma and W. J. Rappel: Phys. Rev. E, 53 (1996), R3017.

27) A. Karma and W. J. Rappel: Phys. Rev. E, 57 (1998), 4323.

$28)$ B. Echebarria, R. Folch, A. Karma and M. Plapp: Phys. Rev. E, 70 (2004), 061604.

29) M. Ohno and K. Matsuura: Phys, Rev. E, 79 (2009), 031603.

30) M. Ohno: Phys. Rev. E, 86 (2012), 051603.

31) M. Ohno, T. Takaki and Y. Shibuta: Phys. Rev. E, 93 (2016), 012802.

32) Y. Shibuta, K. Oguchi and M. Ohno: Scr. Mater., 86 (2014), 20.

33) Y. Shibuta, K. Oguchi, T. Takaki and M. Ohno: Sci. Rep., 5 (2015), 13534.

34) Y. Shibuta, S. Sakane, T. Takaki and M. Ohno: Acta Mater., 105 (2016), 328.

35) T. Takaki, R. Rojas, M. Ohno, T. Shimokawabe and T. Aoki: IOP Conf. Ser., Mater. Sci. Eng., 84 (2015), 012066.

36) S. Sakane, T. Takaki, M. Ohno, T. Shimokawabe and T. Aoki: IOP Conf. Ser., Mater. Sci. Eng., 84 (2015), 012063.

37) A. Yamanaka, T. Aoki, S. Ogawa and T. Takaki: J. Cryst. Growth, 318 (2011), 40.

38) T. Shimokawabe, T. Aoki, T. Takaki, A. Yamanaka, A. Nukada, T. Endo, N. Maruyama and S. Matsuoka: Proc. 2011 SC - Int. Conf. High Performance Computing, Networking, Storage and Analysis, IEEE, Piscataway, (2011).

39) H. J. Diepers, D. Ma and I. Steinbach: J. Cryst. Growth, 237-239, (2002), Part 1, 149.

40) T. Takaki, T. Fukuoka and Y. Tomita: J. Cryst. Growth, 283 (2005), 263.

41) W. Kurz and D. J. Fisher: Fundamentals of Solidification, Trans Technol Publications Ltd., Switzerland, (1989).

42) M. Gündüz and J. D. Hunt: Acta Metall, 33 (1985), 1651.

43) M. Gündüz and E. Çadırlı: Mater. Sci. Eng. A, 327 (2002), 167.

44) The Japan Institute of Metals and Materials: Data book of Metal, 4th ed, Maruzen, (2004), (in Japanese). 